\title{
Essential role of ultraviolet radiation in the decrease of corneal endothelial cell density caused by pterygium
}

\author{
$\mathrm{Xia} \mathrm{Li}^{1} \cdot$ Yiqin Dai ${ }^{2} \cdot$ Weiwei $\mathrm{Xu}^{3} \cdot$ Jianjiang $\mathrm{Xu}^{4}$
}

Received: 12 January 2017 / Revised: 11 June 2018 / Accepted: 9 July 2018 / Published online: 29 August 2018

(c) The Royal College of Ophthalmologists 2018

\begin{abstract}
Purpose To investigate the essential role of ultraviolet (UV) radiation in the decrease of corneal endothelial cell density (ECD) caused by pterygium.

Methods In this retrospective controlled study, 76 consecutive patients with unilateral primary pterygium were enrolled from January 2016 to August 2016. The Central corneal ECD was measured in both eyes. Divide the subjects into two subgroups according to the UV exposure time, and the difference of corneal ECD between both eyes was analyzed using independent samples $t$-test. The relationship between the percentage of difference of corneal ECD and the disease duration, the percentage of pterygium to the cornea and the astigmatism value were analyzed.

Results For subjects with longer UV exposure, the average of ECD in pterygium group and control group were, respectively, $2264.83 \pm 349.78$ cells $/ \mathrm{mm}^{2}$ and $2477.16 \pm 300.36 \mathrm{cells} / \mathrm{mm}^{2}(P=0.004)$. For subjects with short UV exposure, the average of ECD in pterygium group and control group were, respectively, $2596.46 \pm 373.24$ cells $/ \mathrm{mm}^{2}$ and $2690.66 \pm 321.34$ cells $/ \mathrm{mm}^{2}(P=0.262)$. The decrease of corneal ECD was positively correlated with the period of the disease $(P=0.035)$. There was no statistical correlation between a decrease in the corneal ECD and the percentage of pterygium to cornea $(R=$ $-0.055, P=0.635)$ and the astigmatism value in the pterygium eye $(R=-0.135, P=0.246)$.

Conclusions UV radiation exposure played a significant role in the effect of pterygium decreasing the corneal ECD.
\end{abstract}

\section{Introduction}

Pterygium is a wing-shaped neoformation characterized as overgrowth of abnormal conjunctiva onto the cornea and is

Electronic supplementary material The online version of this article (https://doi.org/10.1038/s41433-018-0188-y) contains supplementary material, which is available to authorized users.

Jianjiang Xu

eentjianjiangxu@163.com

1 Department of Ophthalmology and Visual Science, Shanghai Medical College, Eye, Ear, Nose and Throat Hospital, Fudan University, Shanghai, China

2 Key Laboratory of Myopia of State Health Ministry and Key Laboratory of Visual Impairment and Restoration of Shanghai, Eye, Ear, Nose and Throat Hospital, Fudan University, Shanghai, China

3 Shanghai Heping Ophthalmological Hospital, Shanghai, China

4 Department of Ophthalmology and Visual Science, Shanghai Medical College, Eye, Ear, Nose and Throat Hospital, Fudan University, No. 83 Fenyang Road, 200031 Shanghai, China composed of epithelium and fibrovascular connective tissue [1].

Pterygium can influence the structure and function of the ocular surface. When the extension to the cornea exceeds $2.2 \mathrm{~mm}$, obvious corneal astigmatism occurs [2], and visual function would be apparently impaired. Schirmer's test and tear film break-up time are known to be significantly reduced in cases of pterygium indicating the inadequacy of tear film in these patients [3-5].

Pterygium were reported to induce changes much deeper and to result in a decrease in the corneal endothelial cell density (ECD) [6, 7]. As to the mechanism, it is still unclear so far. It was speculated that the damage to the corneal endothelium may be associated with the secretion of some inflammatory factors or the mechanical trauma induced by the abnormal deformation of the cornea caused by pterygium during eye movement [8, 9].

Besides, another essential factor which cannot be neglected was the ultraviolet (UV) radiation. Chronic exposure to UV radiation was found to be the leading factor in promoting the development of pterygium [10, 11]. Meanwhile, the corneal endothelial cells are very 
susceptible to UV radiation [12-14]. Therefore, we supposed that excessive UV exposure may partly account for the decrease of corneal ECD in pterygium eyes. Current researches were unable to confirm the influence of UV exposure on the decrease of ECD [14, 15]. Therefore, we designed a study to investigate the influence of UV radiation on the corneal endothelial cells in the eye with pterygium.

\section{Materials and methods}

This study followed the tenets of the Declaration of Helsinki and was approved by the ethics committee of Shanghai Heping Ophthalmological hospital. Written informed consent was obtained from all the subjects.

\section{Patients and ophthalmic examination}

The study included 76 subjects who were diagnosed with primary unilateral pterygium from January 2016 to August 2016. The other eye of the same subject was considered as control. Those who had bilateral pterygium, recurrent pterygium, or pseudopterygium; those who had any other ocular diseases other than pterygium; those who had undergone any ocular surgeries were excluded.

Detailed ophthalmic examination and slit-lamp photography (Topcon SL-D Digital Slit-Lamp; Topcon, Tokyo, Japan) were carried out for each subject. A $\times 16$ magnification image was taken for further analysis. The percentage of pterygium to the cornea was calculated by thresholding external photos (ImageJ, National Institutes of Health, Bethesda, MD). Sirius Scheimpflug Analyzer (CSO, Costruzione Strumenti Oftalmici, Florence, Italy) quantified corneal astigmatism value and central corneal thickness (CCT). Simulated K (simK) were obtained from the central 3-mm zone of the corneas including flat and steep $\mathrm{K}$ and astigmatism value (difference between steep and flat Ks). Best-spectacle-corrected visual acuity (BCVA) were measured using a Snell eye chart at a distance of $5 \mathrm{~m}$. The Snellen fraction was then converted to approximate ETDRS (approxETDRS) letter scores, which then were used for statistical manipulations using the strategy proposed by Ninel Z [16].

\section{UV exposure measurement}

Measures of potential UV exposure included latitude, daily UV exposure, and sun protection [17-19]. Items on the surveys were from the core skin cancer prevention items for adults [18] (see as appendix) and those which were not associated with the ocular UV exposure or protection were deleted. Usual solar UV exposure was assessed by asking the average number of hours spent outdoors between 10 a. $\mathrm{m}$. and 4 p.m. during the summer on weekdays and on weekends. A weekly average number of daily hours of sun exposure was computed by multiplying the weekday average by 5 , the weekend average by 2 , and dividing by 7 . The group 1 contained patients who spent $>1 \mathrm{~h}$ outdoors per day between 10 a.m. to 4 p.m. in the summer (e.g., farmers, fishermen, etc.). The remaining patients were enrolled in the group 2.

The sun protection strategies were assessed by the values assigned based on frequency of use of three sun protection strategies when outside on a sunny day: wearing hats, wearing sunglasses, and seeking shade when outside $(1=$ never/rarely, $2=$ sometimes, $3=$ often and $4=$ always). 'A sun protection composite' score was computed by averaging the values. For individual sun protection practices analysis, responses were divided into two categories: (1) the sun protection composite was $\geq 3$, (2) the sun protection composite was $<3$.

\section{Corneal ECD measurement}

The central corneal ECD was obtained with the Perseus specular microscope (CSO costruzione strumenti oftalmici, Italy). An automatic acquisition was used to bring the device into focus along with a central fixation light, and when the instrument showed a green light, the image was acquired. A specific area is chosen (the machine setting is $0.06-0.09 \mathrm{~mm}^{2}$ ), and the numbers of cells are counted and the ECD is calculated automatically. Only scans with a reliability index greater than $50 \%$ were chosen. Each patient were measured twice and the average was taken as the mean ECD. All procedures were performed by the same technician. The percentage of difference between the pterygium eye and control eye was calculated using the following format: (ECD of control eye- ECD of pterygium eye)/ECD of control eye.

\section{Statistical analysis}

IBM SPSS Statistics 20.0 was used to perform statistical analysis. The Kolmogorov-Smirnov test was applied to all data samples in order to check normality. If the data follow the normal distribution, it was presented as mean $\pm \mathrm{SD}$, otherwise, it was presented as median (lower quartile, upper quartile). Independent samples $t$-test for independent samples was used to determine whether there was a statistically significant difference between the 2 groups regarding normally distributed continuous variables. If a distribution could not be normalized by logarithmic transformation, the Mann-Whitney $U$-test was used. If the data follow the normal distribution, Pearson correlation statistical test was used to estimate the correlation between two groups, 
Table 1 The difference of parameters between pterygium eye and the control eye

\begin{tabular}{llll}
\hline & Pterygium eye & Control eye & $P$ \\
\hline Corneal ECD & & & \\
All the participants & $2417.55 \pm 395.08$ & $2575.48 \pm$ & $0.011^{\mathrm{a}}$ \\
$N=76$ & & 326.19 & \\
Group 1 $(N=41)$ & $2264.83 \pm 349.78$ & $2477.16 \pm$ & $0.004^{\mathrm{a}}$ \\
& & 300.36 & \\
Group 2 $(N=35)$ & $2596.457 \pm$ & $2690.66 \pm$ & $0.262^{\mathrm{a}}$ \\
& 373.24 & 321.34 & \\
Corneal astigmatism & & & \\
All the participants & $1.5(0.8,7.3)$ & $0.7(0.4,1.2)$ & $<0.001^{\mathrm{b}}$ \\
$N=76$ & & & \\
Group 1 $(N=41)$ & $3.5(1.3,10.6)$ & $0.7(0.5,1.0)$ & $<0.001^{\mathrm{b}}$ \\
Group 2 $(N=35)$ & $1.0(0.5,2.4)$ & $1.0(0.3,1.3)$ & $0.202^{\mathrm{b}}$ \\
BCVA & & & \\
All the participants & $71.2 \pm 18.3$ & $76.4 \pm 9.2$ & $0.029^{\mathrm{a}}$ \\
$N=76$ & & & \\
Group 1 $(N=41)$ & $67.1 \pm 23.4$ & $76.8 \pm 10.1$ & $0.018^{\mathrm{a}}$ \\
Group 2 $(N=35)$ & $75.9 \pm 7.3$ & $75.9 \pm 8.0$ & $0.983^{\mathrm{a}}$ \\
CCT $(\mu \mathrm{m})$ & & & \\
All the participants & $543.57 \pm 31.96$ & $534.97 \pm 33.22$ & $<0.001^{\mathrm{a}}$ \\
$N=61$ & & $531.42 \pm 34.78$ & $0.033^{\mathrm{a}}$ \\
Group 1 $(N=33)$ & $541.45 \pm 34.78$ & $539.14 \pm 32.78$ & $0.001^{\mathrm{a}}$ \\
Group 2 $(N=28)$ & $546.07 \pm 28.06$ & & \\
\hline Group 1 & & &
\end{tabular}

Group 1 represents patients with $>1$ h/day UV exposure

Group 2 represents patients with $\leq 1 \mathrm{~h} /$ day UV exposure

Data is presented as the mean $\pm \mathrm{SD}$, otherwise and as median (lower quartile, upper quartile)

${ }^{a}$ Independent-samples $t$-test

${ }^{\text {b}}$ Mann-Whitney $U$-test

otherwise, Spearman correlation statistical test was used. Chi-square test was used to compare difference of the percentage of female between 2 groups. The automatic linear modeling analysis was used to predict the independent factors for ECD loss. All the subjects were divided into 6 age scopes (20-29, 40-49, 50-59, 60-69, 70-79, 80-89) when the automatic linear modeling analysis was performed.

To calculate the sample size with an overall power of $80 \%, P<0.05$ was used as an acceptable significance level. Using a two-tailed test for independent $t$-test for sample size calculation with effect size 0.5 , we calculated that we would need 64 patients for an $80 \%$ power number. In the end, 76 patients were recruited into the study.

\section{Results}

Seventy six patients were enrolled in the study, including 51 female and 25 male subjects. The mean age was $(66.1 \pm 9.1)$ years, ranging from 26 to 82 years. All the patients were from the Hengsha island, which locates at 121 degrees, 2 min east longitude and 31 degrees, 35 min north latitude, and the annual average sunshine is $2200 \mathrm{~h}$. Forty one subjects were enrolled in group 1, and the remaining 35 subjects were enrolled in group 2. In this study, the sun protection composites in group 1 were all less than 3 , which meant the participates had no awareness of UV protection. And the division of subgroups according to the UV exposure time could demonstrate the severity of UV radiation.

Automatic linear modeling analysis showed that there were three factors which could result in the decrease of the corneal ECD: stronger UV exposure $(B=-263.740, P<$ $0.01)$, age $>80$ years $(B=-428.154, P<0.01)$, and eye with pterygium $(B=-157.928, P=0.002)$.

For all the 76 participants, the corneal endothelial cell density of the pterygium eyes was $2417.55 \pm 395.08$ cells/ $\mathrm{mm}^{2}$, while the ECD of the control eyes was $2575.48 \pm$ 326.19 cells $/ \mathrm{mm}^{2}(P=0.011)$. In group 1 , the average of ECD in pterygium group and control group were, respectively, $2264.83 \pm 349.78$ cells $/ \mathrm{mm}^{2}$ and $2477.16 \pm 300.36$ cells $/ \mathrm{mm}^{2}(P=0.004)$. While in group 2 , the average of ECD in pterygium group and control group were, respectively, $2596.46 \pm 373.24$ cells $/ \mathrm{mm}^{2}$ and $2690.66 \pm 321.34$ cells $/ \mathrm{mm}^{2}(P=0.262)$ (Table 1$)$.

The corneal astigmatism value of pterygium eye was higher than that of control eye in those who underwent severe UV exposure $(P<0.001)$, and the BCVA of pterygium eye was lower than that of control eye in those who underwent severe UV exposure $(P=0.018)$. While in those who underwent mild UV exposure, the corneal astigmatism value and BCVA had no difference between the pterygium eye and control eye $(P=0.202, P=0.983)$.

The CCT of 61 patients were gathered two weeks after surgery. Thirty three participants underwent severe UV exposure, and the remaining 28 underwent mild UV exposure. The CCT in pterygium eyes was larger than that in the contralateral eyes in severe UV exposure group $(P<0.001)$, mild UV exposure group $(P=0.033)$ and for all the participants $(P=0.001)$ (Table 1$)$. For both pterygium eyes and control eyes, there was no statistic difference of CCT between group 1 and group $2(P=0.578, P=0.370)$.

There was no statistic difference of age and gender distribution between group 1 and group 2 (Table 2). For both pterygium eyes and control eyes, the corneal ECD in group 1 was lower than that in group $2(P<0.001, P=0.006)$. For the pterygium eyes, the BCVA of group 1 was lower than that in group $2(P=0.028)$, and the corneal astigmatism in group 1 was higher than that in group $2(P<0.001)$ (Table 2).

The time interval that pterygium appeared until the patients referred to the clinic was recorded. Most patients could not provide the exact period of disease, so we divided 
Table 2 The difference of parameters between group 1 and group 2

\begin{tabular}{llll}
\hline & Group 1 $(N=41)$ & Group 2 $(N=35)$ & $P$ \\
\hline Patients age & $43.4 \pm 15.5$ & $51.5 \pm 19.7$ & $0.111^{\mathrm{a}}$ \\
Sex (percentage of female) & $65.9 \%$ & $68.6 \%$ & $0.213^{\mathrm{b}}$ \\
Duration of disease & $40(30,45)$ & $40(30,50)$ & $0.611^{\mathrm{c}}$ \\
ECD of pterygium eyes & $2264.83 \pm 35.78$ & $2596.46 \pm 373.24$ & $<0.001^{\mathrm{a}}$ \\
ECD of control eyes & $2477.16 \pm 30.36$ & $2690.66 \pm 321.34$ & $0.006^{\mathrm{a}}$ \\
BCVA of pterygium eyes & $67.1 \pm 23.4$ & $75.9 \pm 7.3$ & $0.028^{\mathrm{a}}$ \\
BCVA of control eyes & $76.8 \pm 10.1$ & $75.8 \pm 8.0$ & $0.646^{\mathrm{a}}$ \\
UV protection practice score & $1.3(1.0,1.7)$ & $1.3(1.0,1.7)$ & $0.324^{\mathrm{c}}$ \\
UV exposure time & $4.0(3.0,4.0)$ & $0.4(0.3,0.6)$ & $<0.001^{\mathrm{c}}$ \\
Corneal astigmatism vaule in pterygium eyes & $3.5(1.3,10.6)$ & $1.0(0.5,2.4)$ & $<0.001^{\mathrm{c}}$ \\
Percentage of pterygium to the cornea & $0.22 \pm 0.10$ & $0.14 \pm 0.06$ & $<0.001^{\mathrm{a}}$ \\
CCT of the pterygium eye & $541.45 \pm 34.78$ & $546.07 \pm 28.06$ & $0.578^{\mathrm{a}}$ \\
CCT of the control eye & $(N=33)$ & $(N=28)$ & \\
& $531.42 \pm 34.78$ & $539.14 \pm 32.78$ & $0.370^{\mathrm{a}}$ \\
\hline
\end{tabular}

Group 1 represents patients with $>1 \mathrm{~h} /$ day UV exposure

Group 2 represents patients with $\leq 1 \mathrm{~h} /$ day UV exposure

Data is presented as the mean $\pm \mathrm{SD}$, otherwise and as median (lower quartile, upper quartile)

andependent-samples $t$-test

b chi-square test

${ }^{\mathrm{c}}$ Mann-Whitney $U$-test it as $10,20,30,40$, and 50 years for the different time scopes $(0-14,15-24,25-34,35-44,45-54$ years), respectively. There was no difference of the disease course between the two groups (Mann-Whitney $U$-test, $P=$ 0.611). The duration of the disease was not correlated with the percentage of pterygium to the cornea (Spearman correlation statistical test, $R=0.132, P=0.690$ ) or the astigmatism value in pterygium eyes (Spearman correlation statistical test, $R=0.047, P=0.456$ ).

For all the participants, the percentage of difference of ECD between the pterygium eye and control eye was from $-21.96 \%$ to $66.10 \%$, with $5.72 \%$ on average, which was positively correlated with the duration of disease (Spearman correlation statistical test, $R=0.242, P=0.035$ ) (Fig. 1) and negatively correlated with the BCVA of ptetygium eyes (Spearman correlation statistical test, $R=-0.252, P=$ 0.028 ) (Fig. 2). The percentage of pterygium to cornea was from $2.70 \%$ to $45.95 \%$, with $18.10 \%$ on average, and the median astigmatism value was $1.51 \mathrm{D}$, with $0.77 \mathrm{D}$ and $7.35 \mathrm{D}$ as the upper and lower quartiles. The decrease of corneal ECD were not correlated with the percentage of pterygium to the cornea (Pearson correlation statistical test, $R=-0.055, P=0.635)$ or the astigmatism value in pterygium eyes (Spearman correlation statistical test, $R=$ $-0.014, P=0.902)$.

The astigmatism value of pterygium eyes was determined with a cutoff value of 2D for the difference between pterygium eye and control eye. Thirty two patients had

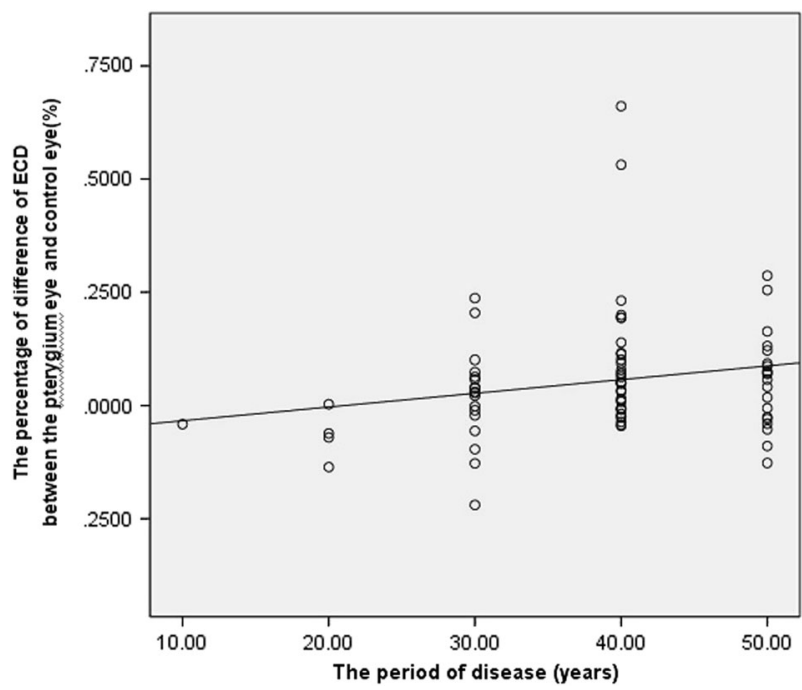

Fig. 1 The period of the disease was positively correlated to the percentage of difference of ECD between the pterygium eye and control eye (Spearman correlation statistical test, $R=0.242, P=0.035$ )

astigmatism value $2 \mathrm{D}$ or more and the range of astigmatism value was from $2.27 \mathrm{D}$ to $37.65 \mathrm{D}$, with a median of $10.82 \mathrm{D}$. The range of decrease in the ECD was from $-21.96 \%$ to $66.1 \%$ with a median of $3.78 \%$. There was no significant correlation between the astigmatism value and the decrease of corneal ECD (Pearson correlation statistical test, $R=$ $0.046, P=0.756$ ). For the other 44 patients with astigmatism value less than $2 \mathrm{D}$, the range of astigmatism value was 


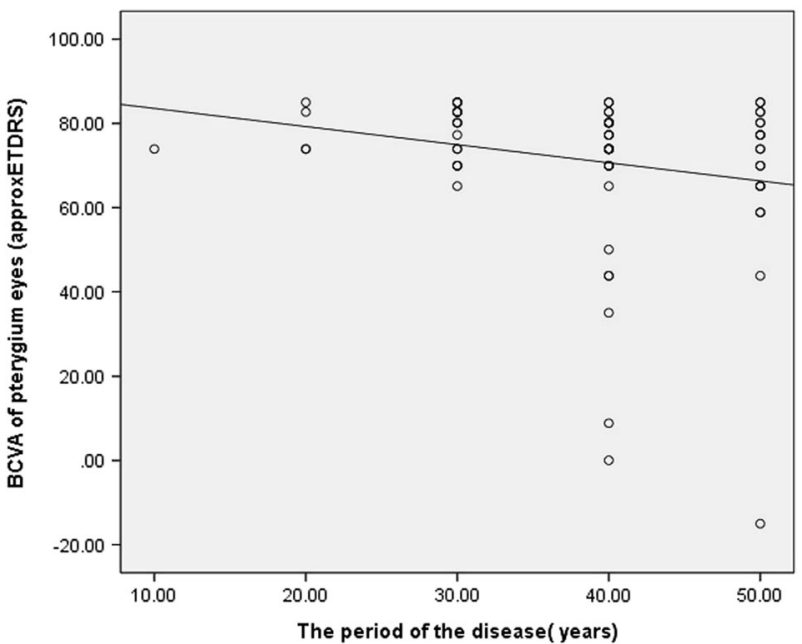

Fig. 2 The period of the disease was negatively correlated with the BCVA of ptetygium eyes (Spearman correlation statistical test, $R=$ $-0.252, P=0.028)$

from $0.08 \mathrm{D}$ to $1.84 \mathrm{D}$ with a median of $0.91 \mathrm{D}$, and the range of decrease in ECD was from $-13.58 \%$ to $53.16 \%$ with a median of $6.85 \%$. There was no significant correlation between the astigmatism value and the decrease of corneal ECD (Pearson correlation statistical test, $R=0.237, P=$ $0.122)$.

\section{Discussion}

In primary unilateral pterygium patients, a decrease in corneal ECD in the pterygium eye compared to the control eye was found in group 1, who underwent a relatively longer UV exposure. While for those who exposed to short UV exposure, the corneal ECD of pterygium eyes did not decrease. As a whole, there was still a decrease in corneal ECD in pterygium eyes compared to control eyes, which agreed with the results of the previous studies [6]. It might be because, in pterygium population, the percentage of people who undergo relatively severe UV radiation is higher. If we studied a different group of subjects who exactly located in the district where the UV radiation is not very serious, and they just exposed to a relatively lower level of UV radiation, the results and conclusions would be different.

The level of UV radiation plays an essential role in the corneal endothelial cells loss caused by pterygium. In this study, for both the pterygium eyes and control eyes, the corneal ECD was lower in group 1 compared to group 2. Longer UV exposure time resulted in loss of corneal endothelium. The conclusion agreed with many previous studies [12, 13, 20-23]. Because of the anatomy, the corneal endothelial cells were exposed to UV radiation directly. UV irradiation would cause formation of intracellular reactive oxygen species (ROS) [12], a major pathogenesis of pterygium [3, 18, 19, 24-26]. In pterygium tissue, high level of 8-hydroxy-2'-deoxyguanosine (8$\mathrm{OHdG}$ ) were found, which indicated oxidative damage of DNA [24, 25]. Furthermore, a higher level of malondialdehyde and nitric oxide had been found in pterygium, together with a lower level of antioxidant enzymes, such as Superoxide Dismutase (SOD), glutathione peroxidase, and catalase [24, 25, 27].

In group 1 , as the contralateral eyes were taken as the control group, the difference of corneal ECD between the pterygium eyes and control eyes was caused by the pterygium. In other words, pterygium can cause the loss of corneal endothelial cells. However, it was not always the case. In group 2, the corneal ECD did not decrease statistically in pterygium eyes. It can be inferred that the longer UV exposure time must have initiated other changes in the cornea in pterygium eyes.

So what changes would happen in the cornea in pterygium eyes after exposure to UV radiation? Some biochemical products may be part of the changes $[7,8,28]$. It was observed that in the pterygium eyes, UV-B irradiation induced a significant increase of matrix metalloprotein (MMP) production in the cornea, which further damaged the descemet membrane and endothelial cells [8, 28]. Moreover, UV exposure leads to a significant increase in the production of interleukin-1 (IL-1), interleukin-6 (IL-6), interleukin-8 (IL-8), and tumor necrosis factor alpha (TNFa) in human corneal stroma cells [29]. TNF-a can break down the barrier integrity of corneal endothelium through the activation of p38 MAP kinase and a consequent disruption of the perijunctional actomyosin ring [30, 31].

Although some inflammatory factors such as MMP and TNF-a can be detected in pterygium eyes, without extra secretion caused by UV radiation, the amount of the biochemical substances might not be large enough to cause the loss of the corneal endothelial cells. Therefore, in group 2, the ptergium did not cause loss of the corneal endothelial cells.

The BCVA of ptergium eyes in group 1 was worse than that in group 2, while the BCVA of control eyes had no statistical difference between the two groups. The percentage of the pterygium to the cornea and astigmatism value in group 1 was larger than that in group 2. The longer UV exposure resulted in the larger size of pterygium. The ptergyium can induce a significant amount of regular and irregular astigmatism in proportion to its size [32]. This can explain the worse BCVA in group 1.

CCT of pterygium eye was found thicker than that of control eye in both group 1 and group 2. Considering that the ECD of the pterygium eyes in severe UV radiation group was still $2264.83 \pm 349.78 / \mathrm{mm}^{2}$, much higher than $800 / \mathrm{mm}^{2}$, in which level, the patients would suffer from 
corneal decompensation combined corneal edema [33]. The difference could not be explained by the difference of ECD. This conclusion was in accordance with the result that even though the ECD of both pterygium eye and control eye in severe UV radiation group was lower than that in mild UV radiation group, no statistic difference of CCT was found between the two groups. Because the size of pterygium in some patients was so large that it was unable to measure the CCT correctly before surgery, we evaluated the CCT two weeks after surgery. Previous research demonstrated that the CCT was not affected by the existence of pterygium [34]. The difference of CCT between pterygium eye and control eye might be ascribed to the factors associated with pterygium surgery and the following alteration of the cornea after surgery, which need further clinical and experimental research.

In one previous study, the arc welders' chronic exposure to the ultraviolet radiation did not cause obvious damage to the corneal endothelium cells [14]. Just as the author concluded it indicated that the UV exposure levels may be below the threshold which would cause damage to the corneal endothelial cells because of their habitual eye protection. While for the study of the effect of UVA on corneal endothelial cells [15], human endothelial cells remained unaffected after directly being irradiated with the clinical dosage for cross-linking. One possible explanation is that when light beam irradiated from the ocular surface, peripheral light focusing (PLF) enlarges the UV energy. Though little direct UV light strikes the ocular surface, intense beam crosses the anterior chamber with PLF, which enables the UV circumvented the anterior layer of corneal and invade the deep cornea, and then damaged the corneal endothelial cells [35]. Moreover, corneal endothelial cells are more sensitive to UVB compared to UVA [36]. Taken together, without PLF, the direct irradiation of UVA, a relatively less harmful rays could not damage the corneal endothelium.

We found a positive correlation between the duration of the disease and the decrease of ECD, which could play a role in determining when surgical intervention is required. Different from Hsu's results [13], we did not find any correlation with the astigmatism and the percentage of pterygium to the cornea. We supposed that the difference between our results and the results of Hsu might be resulted from the different samples. Just as demonstrated in the results part, in our chosen sample, the percentage to the cornea and astigmatism was not correlated with the disease course. Maybe, it was not the case of the participants of Hsu in which there might be some correlation of the disease course with the percentage of pterygium to the cornea and astigmatism. Of course, it was just an assumption and one possible explanation, because professor Hsu did not provide the information of disease duration.
One limitation of our study was that we did not perform the assessment of dry eye disease (DED). Now that DED can be a consequence of pterygium [3-5], and DED can also lead to decreased corneal ECD [37, 38]. So we can not eliminate the possibility that DED may partly account for the loss of corneal endothelial cells.

In conclusion, pterygium and exposure to the UV radiation together will result in a decrease in corneal ECD. It is crucial for the pterygium patients to take anti-UV measures to protect the corneal endothelial cells. The result of the study showed the longer period of disease would result in lower corneal ECD in pterygium and for those who had longer UV exposure time and longer duration of disease suffered worse BCVA. Therefore, it is beneficial for them to have surgery earlier.

\section{Summary}

\section{What was known before}

- Pterygium is related to a decrease in corneal endothelial cell density. However, UV radiation were not taken into consideration.

\section{What this study adds}

- A well validated method was used to assess the UV radiation in the effect on the decrease of corneal ECD. We found that pterygium together with UV radiation would result in the damage of corneal endothelial cells and only pterygium itself was not the sufficient condition to cause the loss of corneal endothelial cells.

\section{Compliance with ethical standards}

Conflict of interest The authors declare that they have no conflict of interest.

\section{References}

1. Nejima R, Masuda A, Minami K, Mori Y, Hasegawa Y, Miyata $\mathrm{K}$. Topographic changes after excision surgery of primary pterygia and the effect of pterygium size on topograpic restoration. Eye Contact Lens. 2015;41:58-63.

2. Mohammad-Salih PA, Sharif AF. Analysis of pterygium size and induced corneal astigmatism. Cornea. 2008;27:434-8.

3. Ishioka M, Shimmura S, Yagi Y, Tsubota K. Pterygium and dry eye. Ophthalmologica. 2001;215:209-11.

4. Türkyılmaz K, Oner V, Sevim MŞ, Kurt A, Sekeryapan B, Durmuş M. Effect of pterygium surgery on tear osmolarity. J Ophthalmol. 2013;2013:863498.

5. Rajiv, Mithal S, Sood AK. Pterygium and dry eye-a clinical correlation. Indian J Ophthalmol. 1991;39:15-6. 
6. Hsu MY, Lee HN, Liang CY, Wei LC, Wang CY, Lin KH, et al. Pterygium is related to a decrease in corneal endothelial cell density. Cornea. 2014;33:712-5.

7. Mootha VV, Pingree M, Jaramillo J. Pterygia with deep corneal changes. Cornea. 2004;23:635-8.

8. Dushku N, John MK, Schultz GS, Reid TW. Pterygia pathogenesis: corneal invasion by matrix metalloproteinase expressing altered limbal epithelial basal cells. Arch Ophthalmol. 2001;119:695-706.

9. Yang SF, Lin CY, Yang PY, Chao SC, Ye YZ, Hu DN. Increased expression of gelatinase (MMP-2 and MMP-9) in pterygia and pterygium fibroblasts with disease progression and activation of protein kinase C. Invest Ophthalmol Vis Sci. 2009;50:4588-96.

10. Moran DJ, Hollows FC. Pterygium and ultraviolet radiation: a positive correlation. Br J Ophthalmol. 1984;68:343-6.

11. Bradley JC, Yang W, Bradley RH, Reid TW, Schwab IR. The science of pterygia. Br J Ophthalmol. 2010;94:815-20.

12. Shimmura S, Suematsu M, Shimoyama M, Tsubota K, Oguchi Y, Ishimura Y. Subthreshold UV radiation-induced peroxideformation incultured corneal epithelial cells: the protective effects of lactoferrin. Exp Eye Res. 1996;63:519-26.

13. Cadet J, Gentner NE, Rozga B, Paterson MC. Rapid quantitation of ultraviolet-induced thymine-containing dimers in human cell DNA by reversed-phase high-performance liquid chromatography. J Chromatogr. 1983;280:99-108.

14. Oblak E, Doughty MJ. Chronic exposure to the ultraviolet radiation levels from arc welding does not result in obvious damage to the human corneal endothelium. Photochem Photobiol Sci. 2002;1:857-64.

15. Mooren P, Gobin L, Bostan N, Wouters K, Zakaria N, Mathysen DG, et al. Evaluation of UVA cytotoxicity for human endothelium in an ex vivo corneal cross-linking experimental setting. J Refract Surg. 2016;32:41-6.

16. Gregori NZ, Feuer W, Rosenfeld PJ. Novel method for analyzing snellen visual acuity measurements. Retina. 2010;30:1046-50.

17. Glenn BA, Lin T, Chang LC, Okada A, Wong WK, Glanz K, et al. Sun protection practices and sun exposure among children with a parental history of melanoma. Cancer Epidemiol Biomark Prev. 2015;24:169-77.

18. Glanz K, Yaroch AL, Dancel M, Saraiya M, Crane LA, Buller $\mathrm{DB}$, et al. Measures of sun exposure and sun protection practices for behavioral and epidemiologic research. Arch Dermatol. 2008;144:217-22.

19. Sekelj S, Dekaris I, Kondza-Krstonijević E, Gabrić N, Predović J, Mitrović S. Ultraviolet light and pterygium. Coll Antropol. 2007;31(Suppl 1):45-7.

20. Liu C, Vojnovic D, Kochevar IE, Jurkunas UV. UV-A irradiation activates Nrf2-regulated antioxidant defense and induces p53/ caspase3-dependent apoptosis in corneal endothelial cells. Invest Ophthalmol Vis Sci. 2016;57:2319-27.

21. Liu C, Chen Y, Kochevar IE, Jurkunas UV. Decreased DJ-1 leads to impaired Nrf2-regulated antioxidant defense and increased UVA-induced apoptosis in corneal endothelial cells. Invest Ophthalmol Vis Sci. 2014;55:5551-60.
22. Serbecic N, Beutelspacher SC. Indoleamine 2,3-dioxygenase protects corneal endothelial cells from UV mediated damage. Exp Eye Res. 2006;82:416-26.

23. Cejková J, Stípek S, Crkovská J, Ardan T, Midelfart A. Reactive oxygen species (ROS)-generating oxidases in the normal rabbit cornea and their involvement in the corneal damage evoked by UVB rays. Histol Histopathol. 2001;16:523-33.

24. Balci M, Şahin S, Mutlu FM, Yağci R, Karanci P, Yildiz M. Investigation of oxidative stress in pterygium tissue. Mol Vis. 2011;9:443-7.

25. Tsai YY, Cheng YW, Lee H, Tsai FJ, Tseng SH, Lin CL, et al. Oxidative DNA damage in pterygium. Mol Vis. 2005;11:71-5.

26. Perra MT, Maxia C, Corbu A, Minerba L, Demurtas P, Colombari R, et al. Oxidative stress in pterygium: relationship between p53 and 8-hydroxydeoxyguanosine. Mol Vis. 2006; $12: 1136-42$.

27. Kau HC, Tsai CC, Lee CF, Kao SC, Hsu WM, Liu JH, et al. Increased oxidative DNA damage,8-hydroxydeoxy-guanosine in human pterygium. Eye. 2006;20:826-31.

28. Koźák I, Klisenbauer D, Juhás T. UV-B induced production of MMP-2 and MMP-9 in human corneal cells. Physiol Res. 2003;52:229-34.

29. Kennedy M, Kim KH, Harten B, Brown J, Planck S, Meshul C, et al. Ultraviolet irradiation induces the production of multiple cytokines by human corneal cells. Invest Ophthalmol Vis Sci. 1997;38:2483-91.

30. Shivanna M, Srinivas SP. Microtubule stabilization opposes the (TNF-alpha)-induced loss in the barrier integrity of corneal endothelium. Exp Eye Res. 2009;89:950-9.

31. Rajashekhar G, Shivanna M, Kompella UB, Wang Y, Srinivas SP. Role of MMP-9 in the breakdown of barrier integrity of the corneal endothelium in response to TNF- $\alpha$. Exp Eye Res. 2014;122:77-85

32. Tomidokoro A, Oshika T, Amano S, Eguchi K, Eguchi S. Quantitative analysis of regular and irregular astigmatism induced by pterygium. Cornea. 1999;18:412-5.

33. Bourne WM, McLaren JW. 'Clinical responses of the corneal endothelium'. Exp Eye Res. 2004;78:561-72.

34. Kılıç R, Çomçalı SÜ, Ağadayı A, Polat OA. The effect of pterygium existence on central corneal thickness. Duzce Med J. 2015;17:13-15.

35. Coroneo M. Ultraviolet radiation and the anterior eye. Eye Contact Lens. 2011;37:214-24.

36. Kataria S, Jajoo A, Guruprasad KN. Impact of increasing Ultraviolet-B (UV-B) radiation on photosynthetic processes. J Photochem Photobiol B Biol. 2014;137:55-66.

37. Kheirkhah A, Saboo US, Abud TB, Dohlman TH, Arnoldner MA, Hamrah $\mathrm{P}$, et al. Reduced corneal endothelial cell density in patients with dry eye disease. Am J Ophthalmol. 2015;159:1022-6.

38. Kheirkhah A, Satitpitakul V, Hamrah P, Dana R. Patients with dry eye disease and low subbasal nerve density are at high risk for accelerated corneal endothelial cell loss. Cornea. 2017;36:196-201. 